\title{
Coal seam gas distribution and hydrodynamics of the Sydney
}

\section{Basin, NSW, Australia}

A. BURRA, J. S. ESTERLE AND S. D. GOLDING

School of Earth Sciences, University of Queensland, Queensland 4072, Australia E-Mail: agiburra@gmail.com

\section{SUPPLEMENTARY PAPERS}

Australian Journal of Earth Sciences (2014) 61, 427-453

http://dx.doi.org/10.1080/08120099.2014.912991

Copies of Supplementary Papers may be obtained from the Geological Society of Australia's website (www.gsa.org.au), the Australian Journal of Earth Sciences website (www.ajes.com.au) or from the National Library of Australia's Pandora archive (http://nla.gov.au/nla.arc-25194). 
APPENDIX 1 REPORTS AND DOCUMENTS ACCESSED FOR BOREHOLE DATA AND LOCAL GEOLOGY AND GAS REGIME INTERPRETATION

\begin{tabular}{ll}
\hline Borehole Name & $\begin{array}{l}\text { NSW Department of } \\
\text { Trade and Investment } \\
\text { DIGS report number }\end{array}$ \\
\hline Bibblewindi 1 & GS2001/049 \\
Bibblewindi 9 & GS2007/864 \\
Big Adder Hill 1 & WCR272 \\
Black Springs 1 & GS2010/0442 \\
Blackville 1 & GS2011/0014 \\
Blackville 1 & GS2011/0014 \\
Boomerang Creek 1 & WCR292 \\
Bootleg 10 & WCR206 \\
Bootleg 2A & WCR195 \\
Bootleg 5 & WCR186 \\
Bootleg 6 & WCR202 \\
Bootleg 7 & WCR187 \\
Bootleg 8 & WCR204 \\
Bootleg 9 & WCR205 \\
Bootleg 9 & WCR205 \\
Brawboy 1 & GS2010/0453 \\
Bulga 1 & WCR265 \\
Catherine Hill Bay 1 & GS2012/0180 \\
Cuan 1 & GS2011/0458 \\
Dartbrook 1 & GS2011/0369 \\
Dewhurst 7 & GS2010/0480 \\
Duncans Creek 1 & WCR278 \\
East Dunlop 1 & WCR267 \\
Faulkland 1A & GS2009/0986 \\
Fullerton 2 & GS2011/0521 \\
Goulburn River 1 & WCR289 \\
Hawkesbury & WCR257 \\
Bunnerong 1 & \\
Hawkesbury Eveleigh 1 & WCR258 \\
Hawkesbury & WCR256 \\
Munmorah 3 & \\
Hunter Bulga 1 & GS2013_0260 \\
Hunter Bulga 2 & GS2013_0259 \\
\hline & \\
\hline
\end{tabular}

\begin{tabular}{ll}
\hline Borehole Name & $\begin{array}{l}\text { NSW Department of } \\
\text { Trade and Investment } \\
\text { DIGS report number }\end{array}$ \\
\hline Hunter Corehole 3 & GS2010/0375 \\
Hunter Corehole 5 & GS2010/0432 \\
Hunter Corehole 6 & GS2010/0431 \\
Hunter Coricudgy 1 & WCR245 \\
Hunter Llanillo 1 & WCR244 \\
Hunter Randwick & WCR243 \\
Park 1 & \\
Jilliby 1 & GS2008/0847 \\
Jilliby 2 & GS2008/0849 \\
Knight 1 & GS2004/310, GS2004/177 \\
Lake Goran 1 & GS2009/0982 \\
Llanillo 1 & WCR244 \\
Maison Dieu 1 & GS2010/0441 \\
Monkey Place 1, 2, & GS2011/0610, \\
3, 4 & GS2011/0611 \\
Moonshine 6 & WCR182 \\
North Castlereagh 1 & WCR262 \\
Oakdale 1 & GS2010/0536 \\
Paynes Crossing 1 & GS2010/0430 \\
Pinegrove 1 & GS2004/309, GS2004/176 \\
Riverstone 1 & WCR266, WCR248 \\
Rouchel Rouchel 1 & GS2010/0449 \\
Rouchel Rouchel 2 & GS2010/0446 \\
Roughit 1 & GS2009/0970 \\
Stratford 10 & GS2011/0201 \\
Turnermans 1 & GS2011/0481 \\
Wappinguy 1 & GS2010/0440 \\
Windermere 1, 2, 3, & GS2011/1422, \\
4 GS2011/0726 \\
Windy Hill 1 & WCR271 \\
Wollombi Brook 1 & WCR270 \\
Wybong 1 & WCR264 \\
\hline & \\
\hline &
\end{tabular}




\begin{tabular}{|c|c|c|}
\hline Mine & Report / Map title & Webpage \\
\hline Dendrobium & $\begin{array}{l}\text { Dendrobium Area 3B Longwalls } 9 \text { to } 18 \text {, Subsidence } \\
\text { Management Plan, Plan 3B - Geology and Seam Floor } \\
\text { Contours. }\end{array}$ & $\begin{array}{l}\text { http://www.bhpbilliton.com/home/aboutus/regulatory/Documents/Dendro } \\
\text { bium\%20Subsidence\%20Management\%20Plan\%20Area\%203B/Dendrobium } \\
\text { Area3B SMPPlan3BGeology-A0.pdf }\end{array}$ \\
\hline Dendrobium & $\begin{array}{l}\text { BHP Billiton, Illawarra Coal - Dendrobium Area 3B } \\
\text { Longwalls } 9 \text { to } 18 \text {. Subsidence Predictions and Impact } \\
\text { Assessments for Natural Features and Surface } \\
\text { Infrastructure in Support of the SMP Application. }\end{array}$ & $\begin{array}{l}\text { http://www.bhpbilliton.com/home/aboutus/regulatory/Documents/Dendro } \\
\text { bium\%20Subsidence\%20Management\%20Plan\%20Area\%203B/Attachment } \\
\text { A-MSEC Subsidence Report.pdf }\end{array}$ \\
\hline Dendrobium & $\begin{array}{l}\text { Dendrobium Colliery - Update on outburst issues in the } \\
\text { Wongawilli seam. Presented at Outburst } 2007 \text { conference, } \\
\text { University of Wollongong, } 28 \text { November } 2007 .\end{array}$ & $\begin{array}{l}\text { http://eis.uow.edu.au/outburst/presentations publications/outburst 2007/ } \\
\text { No.\%203\%20Seam\%200utburst\%20Presentation\%2028-11-07.pdf }\end{array}$ \\
\hline West Cliff & $\begin{array}{l}\text { West Cliff Colliery Area } 5 \text { Longwalls } 34 \text { to } 36 \text {, Subsidence } \\
\text { Management Plan Application, Written Report, January } \\
2008 \text {, Volume } 1 / 3 \text {. }\end{array}$ & $\begin{array}{l}\text { http://www.bhpbilliton.com/home/aboutus/regulatory/Documents/WestCli } \\
\text { ffArea5LW34to36SMPWrittenReportandPlans.pdf }\end{array}$ \\
\hline West Cliff & $\begin{array}{l}\text { Factors affecting the drainage of gas from coal and } \\
\text { methods to improve drainage efficiency. D. Black. PhD } \\
\text { Thesis, 2011. University of Wollongong. }\end{array}$ & http://ro.uow.edu.au/theses/3339 \\
\hline Metropolitan & $\begin{array}{l}\text { Metropolitan Coal, Longwalls 20-22, Subsidence } \\
\text { Monitoring Program, March } 2011 .\end{array}$ & $\begin{array}{l}\text { http://www.peabodyenergy.com/mm/files/operations/australia/metrop/m } \\
\text { anagement-plans/Subsidence Monitoring Program.pdf }\end{array}$ \\
\hline Metropolitan & $\begin{array}{l}\text { Metropolitan Coal Project Environmental Assessment, } \\
\text { Appendix A, The prediction of subsidence parameters and } \\
\text { the assessment of mine subsidence impacts on natural } \\
\text { features and surface infrastructure resulting from the } \\
\text { proposed extraction of longwalls } 20 \text { to } 44 \text { at Metropolitan } \\
\text { Colliery in support of a part } 3 \text { A application. (Mine } \\
\text { Subsidence Engineering Consultants, Report number } \\
\text { MSEC285). }\end{array}$ & $\begin{array}{l}\text { http://www.peabodyenergy.com/mm/files/operations/australia/metrop/m } \\
\text { etrop-project/App\%20A.pdf }\end{array}$ \\
\hline \multicolumn{2}{|c|}{ Tahmoor / Tahmoor North } & Confidential unpublished reports on mine gas drainage \\
\hline
\end{tabular}

